\author{
Charles Kiyaga ${ }^{1 \star}$, Helen $\mathrm{H} \mathrm{Lee}^{2}$ and \\ Jean-Pierre Allain ${ }^{3}$ \\ ${ }^{1}$ Central Public Health Laboratories, Ministry of Health \\ Kampala, Uganda \\ 2Diagnostic Development unit, Dept of haematology, \\ University of Cambridge, Cambridge, UK \\ ${ }^{3}$ Department of Haematology, University of Cambridge, \\ Cambridge, UK \\ Dates: Received: 23 April, 2015; Accepted: 26 \\ June, 2015; Published: 29 June, 2015 \\ *Corresponding author: Charles Kiyaga, Central \\ Public Health Laboratories, Ministry of Health \\ Kampala, Uganda, E-mail: ckiyaga@gmail.com \\ www.peertechz.com \\ ISSN: 2455-3786
}

Keywords: EID; Algorithm; Turnaround Time; LTFU; PCR

\section{Review Article \\ Adherence to Early Infant Diagnosis Testing Algorithm, a Challenge to Early Infant Diagnosis Program in Resource Limited Settings of Uganda}

\section{Abreviations}

EID: Early Infant Diagnosis of HIV; PMTCT: Prevention of Mother to Child Transmission of HIV; WHO: World Health Organization; UNAIDS: Joint United Nations Program on AIDS; MOH: Ministry of Health; HIV: Human Immune deficient Virus; AIDS: Acquired Immune deficiency Syndrome; HCT: HIV Counseling and Testing; ART: Anti-Retroviral Therapy; eMTCT: Elimination of Mother to Child Transmission; HC III: Health Center III; HC IV: Health Center IV; ANC: Antenatal Clinic; DBS: Dry Blood Spot; TAT: Turn Around Time; PCR: Polymerase Chain Reaction; DNA: Deoxy-ribose Nucleic Acid; CPHL: Central Public Health Laboratory; HCW: Health Care Worker; MCH: Maternal Child Health Dept; UNICEF: United Nations Children Education Fund; RRH: Regional Referral Hospital; POC: Point of Care; TAT: Turn Around Time; LTFU: Loss To Follow Up; HCF: Health Care Facility; CDC: Centers for Disease Control

\section{Introduction}

Globally, approximately 1.5 million infants are born to HIV infected women each year, majority of whom are not tested until it is too late for optimal antiretroviral therapy (ART) [1] . Despite the increase in effective methods to prevent mother-to-child transmission (PMTCT) of human immunodeficiency virus type 1 (HIV-1), there were an estimated 390,000 new pediatric HIV-1 infections in 2010, majority of which occurred in resource-limited settings [2]. Without treatment, the mortality rate in HIV infected infants can go as high as $40 \%$ by the first birthday and over $50 \%$ by the second birthday [3]. Recent studies have however shown that early HIV diagnosis and prompt ART intervention can reduce infant mortality by $76 \%$ and HIV progression by $75 \%$ [4]. These studies prompted a change in treatment guidelines by the World Health Organization (WHO) recommending initiation of ART in infants as soon as they are diagnosed as HIV infected [5]. However, the entry into care and treatment programs is dependent on early diagnosis.

Unfortunately, simple antibody-based diagnosis of HIV infection in infants is complicated by the passive transfer of maternal antibodies during pregnancy. Therefore, molecular assays such as polymerase chain reaction (PCR) technology are needed to distinguish HIV infected from HIV exposed but uninfected children during the first 1-2 years of life [6-8]. 
However, PCR-based technologies are too complex and expensive for widespread use in resource poor countries where $90 \%$ of exposed infants are found [9]. The other limitation is that PCR-based technologies require complex infrastructure, skilled manpower, stable supply of electricity and other utilities, which are scarce in developing countries, particularly in rural areas [9]. Therefore, testing is limited to centralized laboratories, posing other challenges such as sample transport and long turnaround time [8].

To promote EID in Uganda and other developing countries, WHO and the U.S. Centers for Disease Control and Prevention (CDC) convened a stakeholders meeting in 2006, following which the Amplicor HIV DNA PCR version 1.5 assay was recommended as the best option available for immediate scale-up of EID programs in the majority of African countries [8]. The report also recommended the use of DBS as a preferred sample type. Like many high burden HIV countries in SSA, Uganda responded by initiating EID services in 2007.

The program begun by using eight regional laboratories run by partners and a courier (Posta Uganda) was contracted to facilitate the referral of DBS samples from health facilities to the regional laboratories, and results back to health facilities. However, an incountry program review conducted in 2010 revealed high overhead costs, laboratory inefficiencies, and long turn-around times (TAT) [10].

In order to improve efficiency, lower operational costs, and improve oversight and coordination, the $\mathrm{MOH}$ consolidated the 8-partner run laboratories, to a single centralized laboratory managed by the $\mathrm{MOH}$ and based at the Central Public Health Laboratories (CPHL) [10]. This innovation was later enhanced by the introduction of the national hub-and-spoke system for DBS sample collection and transport from a network of health facilities to a lab hub from where samples were delivered to the central EID laboratory using the Posta Uganda courier services [11].

EID for exposed infants is not a one off test, but a series over time (6wks to 18 months). This therefore required a national testing algorithm to ensure counter checks in testing process. The EID testing algorithm is outlined below and in appendix 3: exposed infants are tested with 1st HIV molecular test (1st PCR) at 6 weeks of age (coinciding with the 1st immunization visit), or at the earliest opportunity thereafter. If the 1st molecular test is positive, the infant should be initiated on ART the day they receive their results, on which day, a repeat sample for confirmatory PCR is collected and sent for retesting. If the 1 st molecular test is negative, a $2^{\text {nd }}$ molecular test $\left(2^{\text {nd }} P C R\right)$ is performed 6 weeks after cessation of breastfeeding (9-18 months). All children who were screened by molecular test should go through an exit rapid anti-HIV test at 18 months irrespective of results of the earlier tests.

Despite this well laid out testing algorithm in operational guidelines and on result forms sent back to the health facility, routine program data suggests poor adherence to the testing algorithm, though this has not been quantified and its impact to the final diagnosis is not yet known. This study was undertaken to assess adherence to the current testing algorithm.

\section{Materials and Methods \\ Study sites and population}

This evaluation focusing on adherence to the testing algorithm was cross-sectional, retrospective and outcome based. The target population was HIV exposed infants tested within Uganda's centralized EID laboratory in the year 2012 at selected study sites. Study sites included 24 health facilities, selected from six health regions in Uganda including: Gulu in northern region, Arua in northwest, Jinja in eastern region, Masaka in central region, Mbarara in southwest, and Fort Portal in Midwest. In each health region, 4 health care facilities (HCF) covering 4 levels of health care system were selected: one regional hospital (level VI), one general hospital (level V), one health center level IV and one health center level III. In total, there were 6 regional hospitals, 6 general hospitals, 6 health center IVs and 6 health center IIIs. Figure 1 shows the map indicating the location of the study sites and the central testing laboratory.

\section{HIV testing}

The molecular tests were performed using COBAS AmpliPrep Taqman Analyzer, manufactured by Roche Diagnostics Ltd. CH6343 Rotkreuz, Switzerland. The rapid HIV tests were done on mothers using the national testing algorithm, which comprises three anti-HIV rapid tests, which include; Alere Determine HIV $1 / 2$ as screening test (Alere Inc., Scarborough, UK), HIV 1/2 Stat-Pak Assay as confirmatory test (Chembio Diagnostic Systems Inc Medford NY, USA), and Uni-Gold Recombigen HIV 1/2 (Trinity Biotech PLC, Cowicklow, Rep Ireland), as the tie-breaker. These 3 test strips are used in series. The $1^{\text {st }}$ test is the Alere Determine, should the results be positive, it is then retested with Stat-Pak for confirmation. Should the results of Determine and Stat-pack disagree, then Uni-Gold Recombigen would be used as a tie-breaker.

\section{Data collection and analysis}

We designed a data collection template in which we entered patient identification information, testing and follow-up information through the 18 months testing period. The primary data collection tool was the exposed infants' register, from which we captured patient identification and testing information. In case there were gaps, the clinical chart complemented this register. The dispatch form was also checked to fill in any missing information or where there were inconsistencies. The HIV counseling and testing (HCT) register was used to check for the 18 month HIV rapid test results and the pre ART and ART registers were used to check for treatment initiation. From the collection template, data was entered into an Excel spreadsheet developed for this study. It was then cleaned and imported into an access database for analysis. The major study limitation was the incompleteness of data, so some analyses could not be conducted.

\section{Ethical consideration}

The study received IRB approval and also approval by the Uganda National Council of Science and Technology. Since we used retrospective data, there was no direct interaction with patients. However to ensure confidentiality, study IDs were entered into the data collection template instead of patient names. 


\section{Adherence to EID Testing Algorithm study sites}

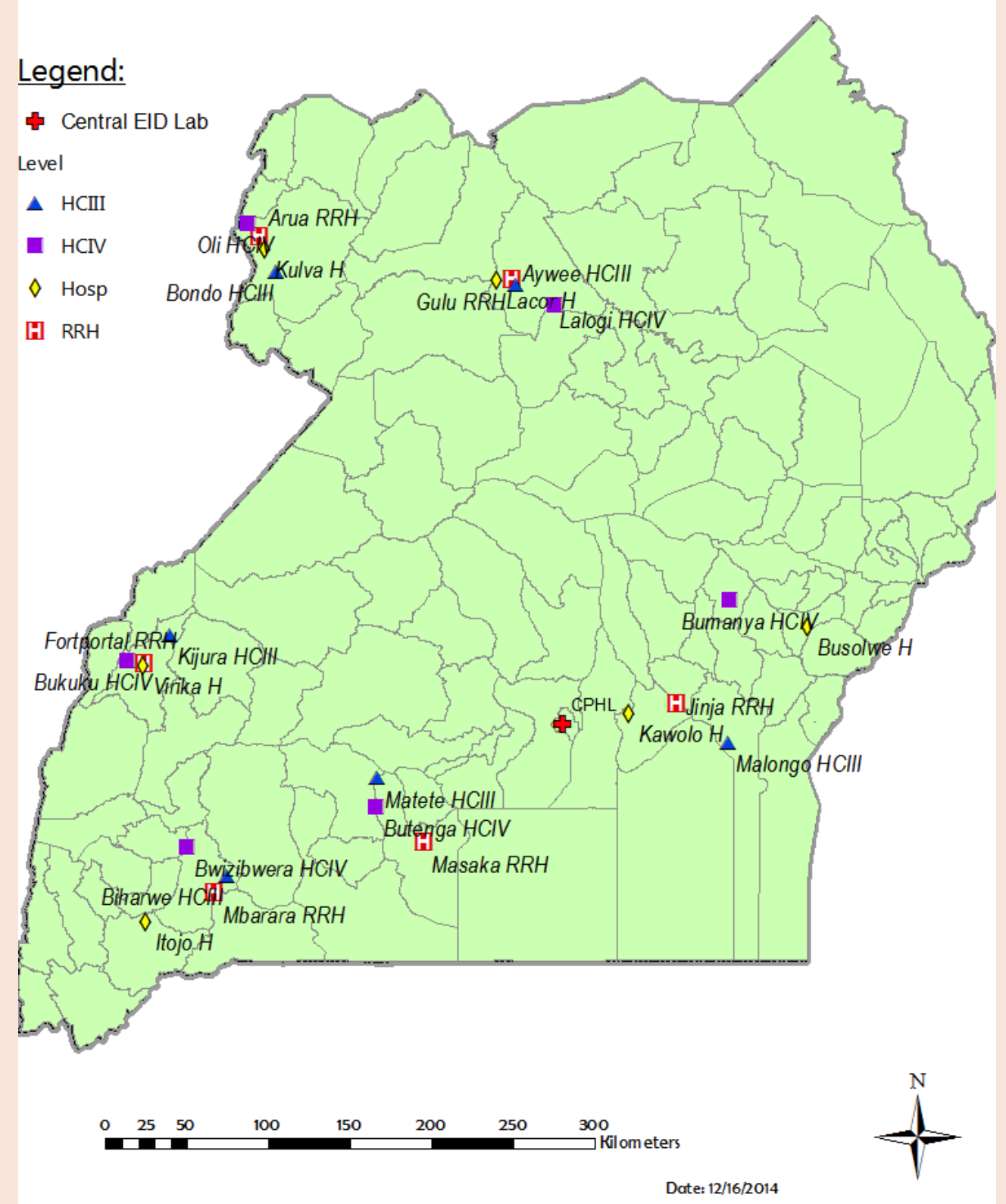

Figure 1: Map of Uganda showing the location of the study sites.

The red square with $\mathrm{H}$ represents regional referral hospital, the yellow diamond the general hospital, the magenta square Health care facility level IV and the blue triangle the health care facility level III. The Red Cross indicates the central public health laboratory (CPHL) where central molecular testing was performed.

\section{Results}

The 24 selected facilities covered the entire tier of the health system, stretching from health center III that is at sub county level to regional referral hospitals, which are at regional or provincial level. The selected health regions were fairly representative of the country (Figure 1). The data is presented in a consolidated form (Figure 2).
From the initial 4427 infants entered in the study, 206 (4.7\%) were excluded for lack of results. Lack of results was due to one of the following; either samples were poorly collected and thus the lab requested for another samples, which was never sent, or samples were sent to the laboratory without any documentation and could therefore not be run by the lab, neither trace where they came from, or results were sent back and given to the patient without being 


\section{Adherence to Testing Algorithm Flow Chart for the 24 Study Sites}

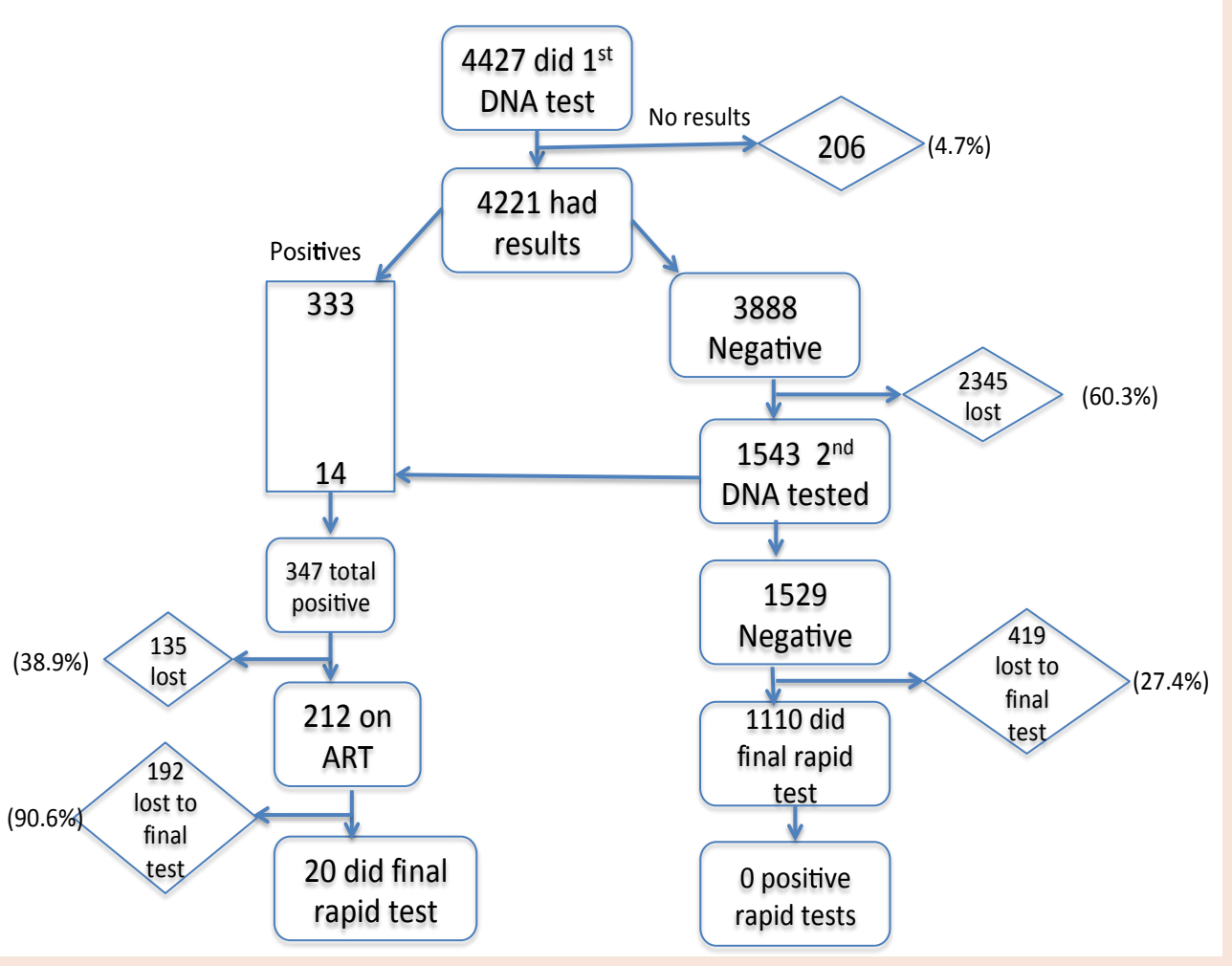

Figure 2: Flow chart that shows the key data that was collected from all the 24 study sites.

Consolidated data collected from all 24 sites, irrespective of health care facility grading. Number of LTFU and \% are indicated at each step in a diamond. Refer to appendix 1 for more details.

entered anywhere at the facilities, therefore the research team found no record at the facility. A total of 4221 infants had results and were therefore included in the study. Table 1 represents the distribution between regions and health care facility levels. The northwest had the lowest number of infants, because it is also a low HIV prevalence region.

In order to critically analyze the data according to EID testing algorithm, three major testing and attrition time ranges were selected: 1) from $1^{\text {st }}$ molecular test to final anti-HIV rapid test for all tested infants, 2) from $1^{\text {st }}$ molecular test to $2^{\text {nd }}$ molecular test for infants who tested negative at the $1^{\text {st }}$ molecular test but still breastfeeding and 3) from receipt of HIV positive results to ART initiation. To better understand the factors associated with loss to follow-up (LTFU) at these time points, TAT of test results associated with each time point was collected.

The age range for $1^{\text {st }}$ molecular test was 0.2 months to 22 months with a median of 2 months and an average of 3.5 months. The age range for $2^{\text {nd }}$ molecular test was 1 month to 24 months with a median of 13.5 months and an average of 12.6 months. The age range for final rapid test was 2.5 months to 36 months with a median of 18 months and an average of 18.6 months.

Figure 2 shows that the prevalence of HIV infection in Ugandan
EID program at $1^{\text {st }}$ molecular test was $7.9 \%$ at an average age of 3.5 months (14 weeks). It further increased to $8.2 \%$ after $2^{\text {nd }}$ molecular test at an average age of 12.6 months (54 weeks). However, this minor increase related to $2^{\text {nd }}$ molecular test does not reflect epidemiology, since $60.3 \%$ of infants were LTFU between $1^{\text {st }}$ and $2^{\text {nd }}$ molecular testing.

Of the total 4221 infants who were tested and had $1^{\text {st }}$ molecular test results, 2976 (70.5\%) caretakers collected their infants' results, representing a loss of $29.5 \%$ and 1130 (26.8\%) were brought back for the final rapid test, representing a LTFU of $62 \%$. From the total 4221 infants who had a $1^{\text {st }}$ molecular test, a cumulative loss of $73.2 \%$ was suffered between the $1^{\text {st }}$ molecular test and the final rapid antibody test. In other words, only $26.8 \%$ adhered to the testing algorithm between $1^{\text {st }}$ molecular test and final rapid test.

From Figure 2, 3888 infants tested negative at the $1^{\text {st }}$ molecular test, and thus should have had a $2^{\text {nd }}$ molecular test. However, of these, only 2645 caretakers collected their infants' results for the $1^{\text {st }}$ molecular test, representing 32\% LTFU. Of the 2645 who collected $1^{\text {st }}$ molecular test results $1543(58.3 \%)$ did a $2^{\text {nd }}$ molecular test, representing $41.7 \%$ LTFU. Of the 1543 who did the $2^{\text {nd }}$ molecular test, $1108(71.8 \%)$ collected results, representing 28.2\% LFTU. Overall, there was a cumulative loss of $71.5 \%$ among infants who had tested 
negative at the $1^{\text {st }}$ molecular test. In other words, only $28.5 \%$ of those who were negative at $1^{\text {st }}$ PCR adhered to the testing algorithm.

From Figure 2, on the infected infants' side, the vast majority of infections were identified by the first molecular test. However, when extrapolating the further infections identified in $40 \%$ of the population tested by second molecular test, a total of 35 new infections were predicted, reaching a total prevalence of HIV infections to $8.7 \%$. Here again, 258 caretakers $(74.4 \%)$ collected their test results, representing $25.6 \%$ LTFU. Of the 258 caretakers who collected results, 212 (82.2\%) were initiated on ART, representing 17.8\% LTFU. Overall, the cumulative LTFU for HIV positive infants stood at 38.9\% (Figure 2). Unsurprisingly, LTFU related to caretakers' attention to infants' health was significantly higher in children identified as non-infected at the first molecular test step $(\mathrm{P}<0.0001)$.

The next step of this analysis was to examine the TAT at each of the three critical steps in the testing algorithm at different HCF levels. We defined TAT for the $1^{\text {st }}$ molecular test as the time elapsed between sample collection from infant to caretaker receiving results. Table 2 shows the distribution of TAT for $1^{\text {st }}$ molecular test between levels of health facilities and regions where they are located. These results indicate that distance from the testing laboratory did not have a major influence on TAT. This is seen from the fact that health facilities from the eastern region that had the shortest distance from the central laboratory $(60 \mathrm{~km})$ had longer TAT than facilities from the northwestern region that had the longest distance from the lab $(504 \mathrm{~km})$ and yet had the shortest TAT.

Table 3 shows the distribution of TAT for $2^{\text {nd }}$ molecular test between different health care levels and regions. A key observation from these results is a marked increase of TAT for $2^{\text {nd }}$ molecular test as compared to $1^{\text {st }}$ molecular test at the same health facilities. For example, the average TAT for $1^{\text {st }}$ molecular test in regional hospitals was 45.9 days (Table 2) as compared to $2^{\text {nd }}$ molecular test where the average TAT was 73.6 days (Table 3). The same was true of general hospitals. This further emphasizes that TAT is not necessarily a factor of distance from the central testing lab. Other factors that might influence TAT need to be investigated.

Table 4 shows the distribution of TAT for ART initiation between different levels of health care facilities from the six regions. TAT for ART initiation was quite long for most facilities apart from those in the Midwest, which averaged at only 1.3 days. This shorter time to ART in the Midwest region might be due to the more efficient PMTCT services, related to the 'Save Mothers Give Life' Project established in the region.

Figure 3 shows the time elapsed between sample collection and receipt of results by the client for $1^{\text {st }}$ molecular test (bar 1), between sample collection and receipt of results by the client for the $2^{\text {nd }}$ molecular test (bar 2) and reception of results of first or second PCR and initiation of ART (bar 3) for positive infants at all study sites. The TAT was ranging between 49 and 72 days (mean 60.2d) for the $1^{\text {st }}$ molecular test with regional referral hospitals having the least and other health centers the longest, between 45 and 75 days for the $2 \mathrm{~d}$ molecular test (mean 68.3d) and between 18 and 45 days (mean 25.9d) between a diagnosis of HIV infection and initiation of ART. Therefore, the accumulation or overlap of caretakers' delay to collect results and delay between sampling and availability of results had a major impact on the massive percentage of LTFUs.

In order to understand the impact of TAT to clients collecting their results, we did an analysis for regional referral hospitals

Table 1: Distribution of patients according to region and type of health care facility.

\begin{tabular}{|c|c|c|c|c|c|c|c|}
\hline & Northern & Northwest & Eastern & Central & Southwest & Midwest & Total \\
\hline RRH & 394 & 110 & 327 & 607 & 541 & 492 & 2471 \\
\hline GH & 259 & 28 & 60 & 242 & 89 & 244 & 922 \\
\hline HCF IV & 105 & 41 & 26 & 23 & 174 & 90 & 459 \\
\hline HCF III & 20 & 18 & 62 & 76 & 46 & 147 & 369 \\
\hline Total & 778 & 197 & 475 & 948 & 850 & 973 & 4221 \\
\hline
\end{tabular}

Table 2: Distribution of TAT for 1st PCR Test between levels of health care facilities (HCF) in days.

\begin{tabular}{|l|c|c|c|c|c|}
\hline HFC & $\begin{array}{c}\text { RRH } \\
\text { Level VI }\end{array}$ & $\begin{array}{c}\text { GH } \\
\text { Level V }\end{array}$ & $\begin{array}{c}\text { HFC } \\
\text { level III }\end{array}$ & $\begin{array}{c}\text { HFC } \\
\text { level IV }\end{array}$ & Distance from central lab (km) \\
\hline Northern & 36.7 & 52.4 & 37.0 & 68.4 & 346 \\
\hline Northwest & 42.4 & 39.0 & 43.0 & 44.5 & 67 \\
\hline Eastern & 41.9 & 86 & 112.5 & 60 \\
\hline Central & 49.3 & 42.6 & 65.8 & 38.0 & 130 \\
\hline Southwest & 62.8 & 68.4 & 38.4 & 93,2 & 266 \\
\hline Midwest & 42.5 & 59.1 & 62.4 & 52.6 & 294 \\
\hline Average & 45.9 & 57.9 & 59.9 & 60.6 & 267 \\
\hline
\end{tabular}

By road, the speed of transport is variable ranging between 30 and $70 \mathrm{~km} / \mathrm{hour}$, depending on the region. Results are sent electronically using GSM printers to the hubs which are mainly level VI and V HCF but not in many of level III and IV. 


\begin{tabular}{|c|c|c|c|c|}
\hline HFC & $\begin{array}{c}\text { RRH } \\
\text { level VI }\end{array}$ & $\begin{array}{l}\text { GH GH } \\
\text { level V }\end{array}$ & $\begin{array}{c}\text { HFC } \\
\text { level III }\end{array}$ & $\begin{array}{c}\text { HFC } \\
\text { level IV }\end{array}$ \\
\hline Northern & 72 & 50 & 32 & 33 \\
\hline Northwest & 78 & 60 & 40 & 51.8 \\
\hline Eastern & 60.3 & 91 & 105.3 & 77.6 \\
\hline Central & 68 & 59.5 & 140 & 51 \\
\hline Southwest & 100.8 & 62.5 & 16 & 12.6 \\
\hline Midwest & 62.6 & 109.4 & 65.6 & 50 \\
\hline Average & 73.6 & 72 & 66.5 & 46 \\
\hline
\end{tabular}

Table 4: Distribution of TAT for ART initiation between levels of health care facilities (HCF) in days.

\begin{tabular}{|l|c|c|c|c|}
\hline HFC & $\begin{array}{c}\text { RRH } \\
\text { level VI }\end{array}$ & $\begin{array}{c}\text { GH } \\
\text { level V }\end{array}$ & $\begin{array}{c}\text { HFC } \\
\text { level III }\end{array}$ & $\begin{array}{c}\text { HFC } \\
\text { level IV }\end{array}$ \\
\hline Northern & 23 & 21 & - & 33 \\
\hline Northwest & 20 & - & - & 1 \\
\hline Eastern & 21 & 58 & 18 & - \\
\hline Central & 15 & 12 & 68 & 221 \\
\hline Southwest & 47 & 30 & 0 & 0 \\
\hline Midwest & 3 & 0 & 0 & 25.7 \\
\hline
\end{tabular}

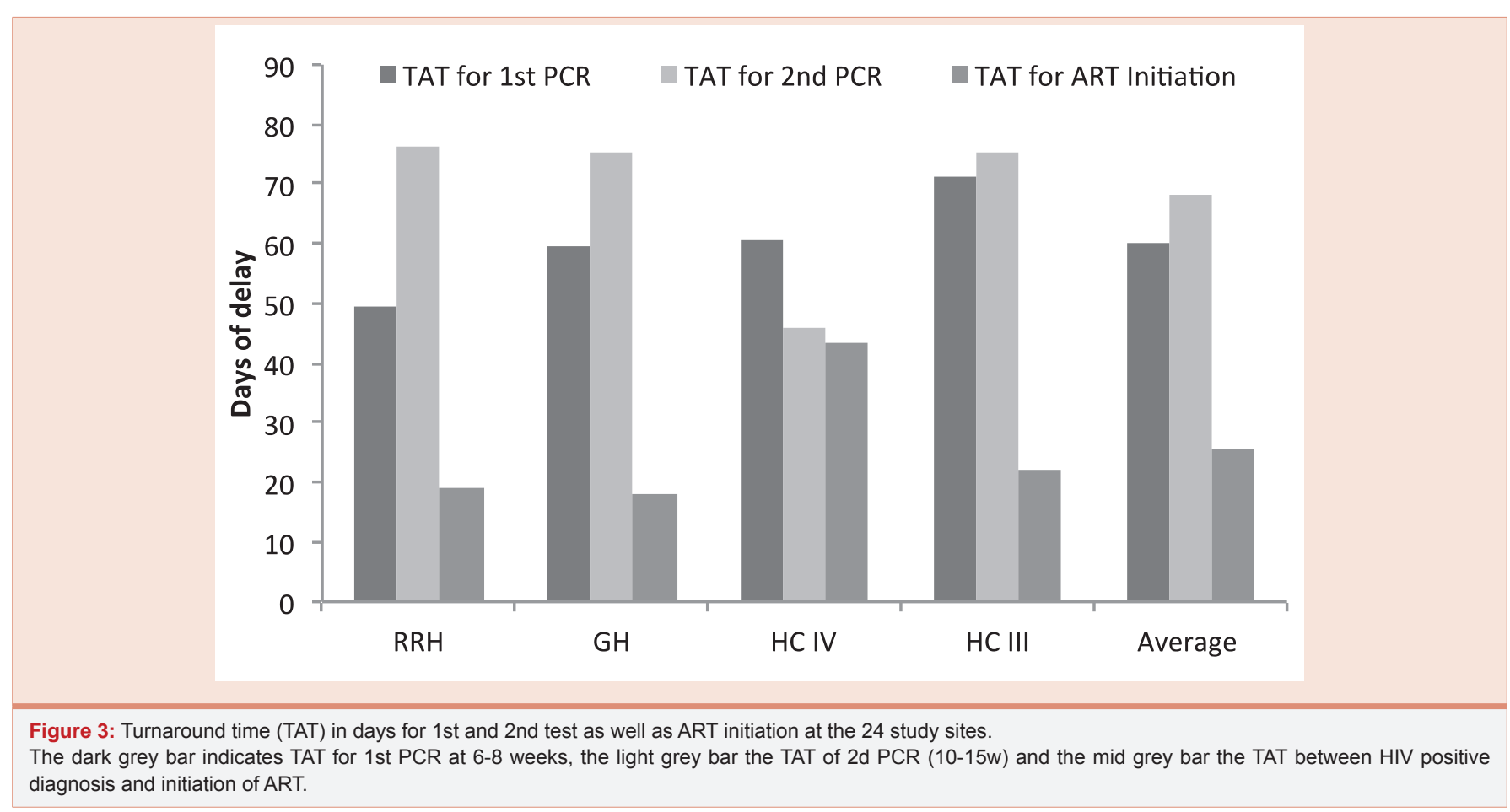

(Figure 4) (Tables 5, 6). This data shows that Fort Portal hospital with an average TAT of 42 days had $81 \%$ of clients who collected their results as compared to an average TAT of 63 days in Mbarara regional hospital with only $64 \%$ caretakers collecting results. Regional hospitals put together have a TAT of 45.9 days and have $72 \%$ of caretakers collecting results as compared to health center IIIs where TAT was 60 days with $62 \%$ caretakers collecting results (Table 7).

\section{Discussion}

The observed HIV positivity of $8.7 \%$ appears similar to the national average by then [12], but has of late gone down to about $5.6 \%$ due to option $\mathrm{B}+$ being offered to HIV infected pregnant and lactating women [13]. The average age at first molecular of 14 weeks was higher compared to the recommended 6 weeks according to the 


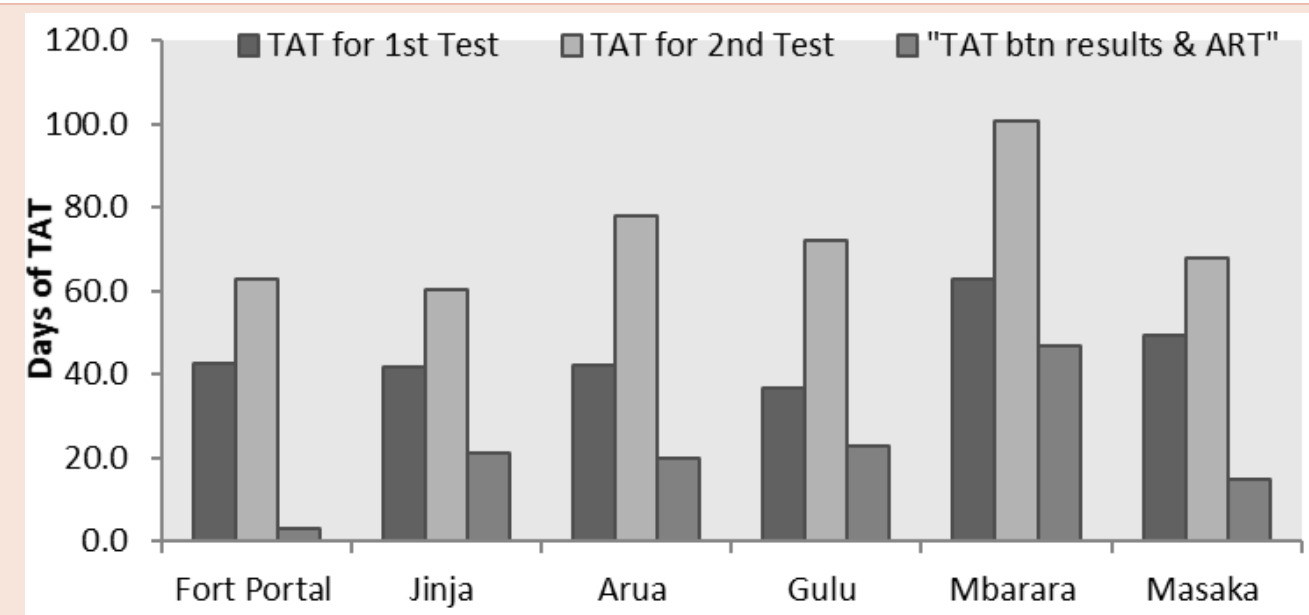

Figure 4: Comparison of turnaround time (TAT) in days for 1st and 2nd test as well as ART initiation between 6 regional referral hospitals. Dark grey bars represent TAT for 1st PCR, light grey bars represents TAT for $2 \mathrm{~d}$ PCR and mid grey bar for rapid test TAT.

Table 5: Distribution of TAT for 1st PCR, 2nd PCR and ART Initiation at Regional Referral Hospitals (RRH).

\begin{tabular}{|c|c|c|c|c|}
\hline RRH Level I & TAT for 1st mol test & TAT for 2 nd mol. test & TAT for ART Initiation & Distance from Central lab (km) \\
\hline Gulu & 36.7 & 72 & 23 & 346 \\
\hline Arua & 42.4 & 78 & 20 & 504 \\
\hline Jinja & 41.9 & 60.3 & 21 & 60 \\
\hline Masaka & 49.3 & 68 & 15 & 130 \\
\hline Mbarara & 62.8 & 100.8 & 47 & 266 \\
\hline Fort Portal & 42.3 & 62.6 & 3 & 294 \\
\hline Average & 45.9 & 73.6 & 21.5 & 267 \\
\hline
\end{tabular}

Table 6: Distribution of TAT for 1st PCR, and proportion who collected 1st PCR Results at Regional Referral Hospitals (RRH)

\begin{tabular}{|l|c|c|}
\hline RRH Level I & TAT for 1st PCR & Proportion who picked 1st PCR Results \\
\hline Gulu & 36.7 & 68.3 \\
\hline Arua & 42.4 & 73 \\
\hline Jinja & 41.9 & 73.7 \\
\hline Masaka & 49.3 & 73 \\
\hline Mbarara & 62.8 & 64.4 \\
\hline Fort Portal & 42.5 & 80.7 \\
\hline Average & 45.9 & 72.2 \\
\hline
\end{tabular}

testing algorithm. The HIV molecular testing was set up at 6 weeks to coincide with the first appointment for vaccination. Our data raises the issue of the connection between HIV testing and vaccination. If they remain linked, it poses a serious issue as to the adherence to the vaccination schedule. If it is not connected, the timing of 6 weeks can be challenged since testing earlier might be an advantage for controlling HIV morbidity in infants and newborn. In any case, the 8-14 weeks for first molecular test is a clear shifting from the algorithm that needs to be corrected given the vulnerability of these infants [4] in whom HIV tends to take a more aggressive mode as compared to adults [3].
Another weakness found with this part of the EID algorithm is that, not all tested infants' caretakers came back to collect their test results. As presented in the result section, the delay by caretakers to collect test results is multifactorial and shared between TAT of test results provided by the central testing laboratory, the appointment system of the clinics which may vary according to HCF level and region and the level of concern of families. According to the data, $29.5 \%$ of those who did a $1^{\text {st }}$ molecular test and $32 \%$ of those who did a $2^{\text {nd }}$ molecular test never came back to collect their results. The system of appointment adopted by the clinics to coincide with vaccination appointments may look justified for the first molecular test appointment using 
Table 7: Distribution of TAT for 1st molecular test, and proportion of caretakers who collected 1st molecular test results at different levels of health facilities.

\begin{tabular}{|l|c|c|}
\hline HF Level & TAT for 1st PCR & Proportion who picked 1st PCR Results \\
\hline RRH & 45.9 & 72.2 \\
\hline GH & 57.9 & 79 \\
\hline HC IV & 60.6 & 68.6 \\
\hline HC III & 59.9 & 62.4 \\
\hline
\end{tabular}

vaccination as an incentive at six weeks of age. However, evidence collected here suggests that the broad range of first molecular test samples collected might reflect a degree of disconnection between HIV and vaccination appointments. Instead of setting appointments at one-month intervals, a direct communication from the clinic to the family by mobile phone as soon as results are received might help the initiation of ART and limit LTFU.

Here we examined one of the three factors that appear the most critical: TAT for test results. One study reported that long TAT was one of the major reasons for failure to collect results [14]. It appears to be longer in the southwest region despite being in the mid distance from Kampala where the central testing laboratory is located (Table 2). Unsurprisingly, regional referral hospitals at level VI had the shortest TAT, with the smallest range between regions (Figure 4). This is likely due to the fact that all regional hospitals are hubs, and therefore receive results faster than non-hub health facilities. Lower HCF which are not hubs don't have access to electronic communication with the central lab, there by relying on bike riders to drop results, which may delay for a couple of days up to 1 week in the event results arrive on a day the rider is not visiting the site. The limited data available does not support that distance between HCF and central laboratory is a significant factor but distance between infants home and HCF might be, although we did not have the data to prove such hypothesis (Table 2). Other factors such as attitude of health workers and clients, lack of a patient follow-up mechanism at the facility, lack of transport for the caretakers, and others should be investigated.

The $2^{\text {nd }}$ molecular test is intended to diagnose postpartum transmission through breastfeeding. To avoid nutrition-related deaths, mothers are encouraged to exclusively breastfeed for the first 6 months, after which they add supplementary feeding up to one year and then go for accelerated weaning [15]. Therefore, $>98 \%$ of HIV infected mothers in Uganda are breastfeeding and the eMTCT agenda through option B+ aims at making breastfeeding safer. Given that background, we expect all HIV positive mothers, who had a noninfected baby at $1^{\text {st }}$ molecular test, to bring the baby back for a second molecular test 6 weeks after cessation of breastfeeding.

From the data collected, 3888 babies tested negative at the $1^{\text {st }}$ molecular test and mothers were thus expected to bring back their babies for $2^{\text {nd }}$ molecular test after breastfeeding (Table 3 ). However, only $1543(39.7 \%)$ brought back their infants for a $2^{\text {nd }}$ molecular test, representing $60.3 \%$ LTFU in total. Of those babies tested, 14 (0.91\%) were found HIV infected and added to the HIV positive cohort eligible for ART. These results are not very different from the $1.4 \%$ postpartum transmission reported in a previous study [16].

The LTFU after the $2^{\text {nd }}$ molecular test was greater in health centers level III and IV than in hospitals (Level V and VI) (Table 4). It is a known fact that most post-partum MTCT occurs through breastfeeding [15], and failure to do a $2^{\text {nd }}$ molecular test after breastfeeding, as stipulated in our testing algorithm, may lead to missed opportunities of early identification and ART initiation [4].

It is surprising to note that only $39.7 \%$ of caretakers brought back the infants to the clinic for a second molecular test after cessation of breastfeeding. It would be expected that having made the effort to come twice already to the clinic would have encouraged them to return a third time to potentially exonerate their child from HIV infection. It is also surprising that TAT for the $2^{\text {nd }}$ molecular test is nearly one month longer than for the $1^{\text {st }}$ molecular test. This is clear from Table 5 where the difference in TAT between $1^{\text {st }}$ and $2^{\text {nd }}$ molecular test is an average of 28 days within regional hospitals. The TAT at the central laboratory should be the same irrespective of the sample collected, and the rest of TAT should have remained the same, because the distances between the lab and the health facility and the health facility and the clients' homes remain the same. However, this indicates that there are other factors in play. The degree of involvement and dedication of the HCF staff might be one of them. In addition, the regular appointments set up for vaccination are no longer a factor after 4-5 months of age and this disconnection might make the clinic connection with the caretakers more problematic. Here again direct contact by mobile phone would seem a legitimate approach to improve the situation.

According to the EID testing and care algorithm; "Any HIV positive child under 2 years of age should be initiated on treatment the same day they get results irrespective of clinical or immunological staging". However, almost $40 \%$ of infants confirmed HIV positive were not initiated on ART. To find patients who are lost to followup can be difficult, costly and inefficient [17], which highlights the need to prevent losses. One option to improve the situation might be to establish better contact between the clinic staff and caretakers by telephone calls. Another probably more efficient option would be point of care (POC) testing, where the test can be performed and results given to the family on the visit day. However POC may solve the problem of returning results to care takers, but issues related to poor ART initiation go beyond return of results, since the data shows that there were some infants who got results but were not initiated on treatment.

According to the testing algorithm, the treatment initiation TAT should be 0 days, meaning that the same day caretakers receive results ART should be initiated. However according to Figure 3, data shows that it takes averagely 18.8 days for regional and general hospitals, and 33 days for health center IIIs and IVs. The shorter interval in HCF level VI and V (18.8 days) than in HCF level III and IV (33 days) 
might be due to lack of capacity for ART initiation at lower health facilities than higher ones [4]. The confidence and experience in such decisions is probably better for senior physicians than lower cadre health workers available in lower level HCF. If health workers were adhering to this part of the testing algorithm, the number of patients who initiate treatment would be equal to the number of infants who collect results.

The National EID testing algorithm stipulates that; "for all exposed infants under 18 months of age tested by a molecular test, the same will be retested by a rapid test after 18 months of age irrespective of the results of the earlier molecular test". This was put there as a counter check, to weed out any miss diagnosis that may have arisen due to human and technical errors in the earlier testing. However, data from this study reveals that, of the 4221 infants tested by $1^{\text {st }}$ molecular assay, only 1130 (26.8\%) ever did the final rapid test. This shows the lack of a system that ensures that the testing algorithm is adhered to. However, no new infection cases were identified through the rapid test $(0 / 1130)$ suggesting a low risk of breastfeeding transmission beyond the first year of life.

A study conducted in Kenya [18] to assess discrepant test results in their EID program presented interesting findings. Over $2.5 \%$ of what was classified as positive and over $1.88 \%$ of what was classified as negative by manual PCR assay, tested otherwise on retesting using the automated assay. False positive and false negative results can arise from clerical errors, contamination, or from limitations of the technology used. It was against this background that this final rapid test was put within the testing algorithm. However, failure to operationalize this part of the testing algorithm leaves the program in a dilemma. We might be having infants on treatment who were wrongly diagnosed and have been committed to lifelong ART despite its deterring effects, discomfort, inconvenience and cost.

\section{Conclusions}

EID programs in resource limited settings face a lot of problems that start with identification of HIV positive mothers during pregnancy through PMTCT, identification of HIV exposed infants during the postnatal period and linking them to testing, retaining infants into the testing and care algorithm and initiating the positive infants on ART. However, this study concentrated on retaining the identified infants into testing and care algorithm and initiating positives onto ART.

The uniqueness of EID is derived from the fact that the testing is not a one off, but a series over a period of time, which facilitates loss, as long as there are no mechanisms of patient follow-up integrated into the testing process.

Results of this study show that it is one thing to have a testing algorithm but another thing to have it adhered to. There is already a discussion within WHO to revise EID testing algorithm by adding a PCR test at birth and a rapid test at 9 months to the existing algorithm. If the current algorithm is poorly adhered to, adding additional tests may not help the situation.

Uganda's EID program still faces challenges of poor retention of infants into the testing and care algorithm and poor ART initiation for the positives. However, these challenges may not be unique to Uganda but might be generalized among resource limited settings

\section{Recommandations}

In view of results from this study demonstrating poor adherence to testing algorithm, similar programs should undertake equivalent assessments.

Before revising the current testing algorithm by adding additional tests, the current algorithm should be strengthened by ensuring it is adhered to.

Since TAT is one of the major causes of LTFU at the different time points, efforts to reduce TAT especially when results reach the facility should be made by alerting clients through telephone calls when results come back.

To improve adherence to the $2^{\text {nd }}$ PCR test and the final rapid test, patients may need to be reminded through telephone calls or other means.

Because of poor adherence to testing algorithm, spot checks should be done to assess discordance, like was done in Kenya by Kageha et al 2012.

More capacity for pediatric ART initiation should be built especially at lower level health facilities, where time to ART initiation was high.

There is need to integrate patient follow-up and care into the EID testing process.

Point of care EID testing when available should be used to complement centralized EID program, especially in hard to reach areas and other sites, which for one reason or another experience excessive TAT.

However, having analyzed the many challenges the current centralized program is facing, it is important to make serious considerations as we think of deploying point of care platforms. Being able to provide results the same day may not mean these results will automatically impact patient care. Just having a POC in itself may not translate into improved patient outcomes [19-24].

\section{Acknowledgement}

I acknowledge the help offered by the study site staff who assisted me during the protracted process of data collection. Thanks also go to the in charges of the study sites, who allowed me access to the site data records, and in most cases dedicated a staff to work with me.

I also acknowledge the support offered by the EID team based at the Central Public Health Laboratories, led by the lab manager Isaac Sewanyana, Chris Okira, Allan Kisakye and Rebecca Nakidde for all the support they offered me during the process of data collection, entry and analysis. Thanks also go to Mr. Aisu Steven the Head of Central Public Health Laboratories for his inspiration and guidance.

Overall thanks goes to Dr. Jane Ruth Aceng the Director General of Health Services who gave me authority letters to the study sites. 


\section{References}

1. Bassett IV, Wang B, Chetty S, Mazibuko M, Bearnot B, et al. (2009) Loss to care and death before antiretroviral therapy in Durban South Africa. J Acquir Immune Defic Syndrome 51: 135-139.

2. WHO (2011) Progress Report 2011: global HIVIAIDS response.

3. Newell ML, Coovadia H, Cortina-Borja M, Rollins N, Gaillard P, et al. (2004) Mortality of infected and un infected infants born to HIV infected mothers in Africa: a pooled analysis. Lancet 364: 1236-1243.

4. Avy Violari, FC.Paed, Mark F Cotton, M.Med, Diana M Gibb, et al. (2008) Early antiretroviral therapy and mortality among HIV infected infants. N Eng J Med 359: 2233.

5. WHO (2008) Towards universal access: Scaling up priority HIV AIDS interventions in the health sector. Geneva Switzerland, World Health Organization.

6. Sherman GG, Cooper PA, Coovadia AH, Puren AJ, Jones SA, Mokhachane $M$, et al. (2005) Polymerase chain reaction for diagnosis of human immunodeficiency virus infection in infancy in low resource settings. Pediatr Infect Dis J 24: 993-997.

7. Creek TL, Sherman GG, Nkengasong J, Lu L, Finkbeiner T, et al. (2007) Infant human immunodefficiency virus diagnosis in resource limited settings: issues, technologies and country experiences. Am J Obstet Gynecol 197 S64-71.

8. W Stevens, Sherman G, Downing R, Parsons LM, Ou CY, et al. (2008) Role of Laboratory in Ensuring Global Access to ARV Treatment for HIV infected Children: Consus Statement on the Performance of Laboratory Assays for Early Infant Diagnosis. Open AIDS J 2: 17-25.

9. Creek TL, Sherman GG, Nkengasong J, Lu L, Finkbeiner T, et al. (2007) Infant human immunodeficiency virus diagnosis in resource-limited settings: issues, technologies, and country experiences. Am J Obstet Gynecol 197: S64 - S71.

10. Kiyaga C, Sendagire H, Joseph E, Grosz J, McConnell I, et al. (2015) Consolidating HIV testing in a Public Health Laboratory for Efficient and Sustainable Early Infant Diagnosis (EID) in Uganda. J Public Health Policy 36: $153-169$

11. Kiyaga C, Sendagire H, Joseph E, McConnell I, Grosz J, et al. (2013) Uganda's New National Laboratory Sample Transport System: A Successful Model for Improving Access to Diagnostic Services for Early Infant HIV Diagnosis and Other Programs. PLoS One 8: e78609.

12. MoH (2011) Uganda AIDS Indicator Survey (UAIS). Kampala Uganda.

13. Mwapasa V, Pro G, Chinkhumba J, Mukaka M, Kobayashi E, et al. (2014) Mother-Infant Pair Clinic and SMS Messaging as Innovative Strategies for
Improving Access to and Retention in eMTCT Care and Option B+ in Malawi: A Cluster Randomized Control Trial. Journal of Acquired Immune Deficiency Syndromes 67: S120-S124.

14. Mugambi ML, Deo S, Kekitiinwa A, Kiyaga C, Singer ME (2013) Do Diagnosis Delays Impact Receipt of Test Results? Evidence from the HIV Early Infant Diagnosis Program in Uganda. PLoS ONE 8: e78891.

15. Rollins NC, Becquet R, Orne-Gliemann J, Phiri S, Hayashi C, et al. (2014) Defining and Analyzing Retention-in-Care Among Pregnant and Breastfeeding HIV-Infected Women: Unpacking the Data to Interpret and Improve PMTCT Outcomes. J Acquir Immune Defic Syndr 67: S150-S156.

16. Njom Nlend AE, Same Ekobo C, Bagfegue Ekani B, Epée Ngoue J, Tetang Ndiang S, et al. (2013) Preventing HIV-1 transmission in breastfed infants in low resource settings: early HIV infection and late postnatal transmission in a routine prevention of mother-to-child transmission program in Yaounde, Cameroon. J Trop Pediatr 59: 387-392.

17. Forster M, Bailey C, Brinkhof MW, Graber C, Boulle A, et al. (2008) Electronic medical record system, data quality and loss to follow up: survey of antiretroviral therapy programs in resource limited settings. Bull World Health Organ 86: 939-947.

18. Kageha S, Okoth V, Kadima S, Vihenda S, Okapesi E, et al. (2012) Discrepant test findings in early infant diagnosis of HIV in a national reference laboratory in Kenya: challenges and opportunities for programs. J Trop Pediatr 58: 247252.

19. Myer L, Wilkinson D, Lombard C, Zuma K, Rotchford K, et al. (2003) Impact of on-site testing for maternal syphilis on treatment delays and treatment rates and perinatal mortality in rural South Africa: a randomized control trial. Sex Transm Infect 79: 208-213.

20. Ansah EK, Narh-Bana S, Epokor M, Akanpigbiam S, Quartey AA, et al. (2010) Rapid testing for malaria in settings where microscopy is available and peripheral clinics where only presumptive treatment is available: a randomised controlled trial in Ghana. BMJ 340: c930.

21. Ntombizodumo B. Mkwanazi, Deven Patel, Marie-Louise Newell, Nigel C. Rollins, A. Coutsoudis, et al. (2008) Rapid testing may not improve uptake of HIV testing and same day results in a rural South African community: a cohort study of 12,000 women. PLoS One 360.

22. Rosen S, Fox MP (2011) Retention in HIV care between testing and treatment in sub-Saharan Africa: a systematic review. PLoS Med 8: e1001056.

23. Jani IV, Sitoe NE, Alfai ER, Chongo PL, Quevedo JI, et al. (2011) Effect of point-of-care CD4 cell count tests on retention of patients and rates of antiretrovi- ral therapy initiation in primary health clinics: an observational cohort study. Lancet 378: 1572-1579.

24. Jain IV, Peter TF (2013) How Point of Care testing could drive innovations in Global health. N Engl J Med 368: 2319-2324.

Copyright: (c) 2015 Kiyaga C, et al. This is an open-access article distributed under the terms of the Creative Commons Attribution License, which permits unrestricted use, distribution, and reproduction in any medium, provided the original author and source are credited. 